\title{
Universal terms for holographic entanglement entropy in noncommutative Yang-Mills theory
}

\author{
Tadahito Nakajima \\ College of Engineering, Nihon University, Fukushima 963-8642, Japan
}

(Received 31 August 2020; accepted 4 March 2021; published 9 April 2021)

\begin{abstract}
In this paper, we derive the universal (cutoff-independent) part of the holographic entanglement entropy in the noncommutative Yang-Mills theory and examine its properties in detail. The behavior of the holographic entanglement entropy as a function of a scale of the system changes drastically between large noncommutativity and small noncommutativity. The strong subadditivity inequality for the entanglement entropies in the noncommutative Yang-Mills theory is modified in large noncommutativity. The behavior of the entropic $c$-function defined by means of the entanglement entropy also changes drastically between large noncommutativity and small noncommutativity. In addition, there is a transition for the entanglement entropy in the noncommutative Yang-Mills theory at finite temperature.
\end{abstract}

DOI: 10.1103/PhysRevD.103.086005

\section{INTRODUCTION}

The noncommutative gauge theories discussed in this paper are gauge theories in which the product of any two fields is given by the Moyal-Weyl product [1,2]

$$
f \star g(x) \equiv f(x) \exp \left(\frac{i}{2} \theta^{\mu \nu} \partial_{\mu} \overrightarrow{\partial_{\nu}}\right) g(x)
$$

where $\theta^{\mu \nu}$ is the deformation parameter. It is well known that these gauge theories naturally arise as low-energy theories of D-branes in a Neveu-Schwarz-Neveu-Schwarz $B$-field background [3-6]. A remarkable phenomenon in these gauge theories is UV/IR mixing [1,2], where the UV and IR degrees of freedom of the theory are mixed in a complicated way. It is very important to deepen the understanding of such quantum effects in both perturbative and nonperturbative approaches.

There exists a holographic description for the strongly coupled noncommutative gauge theories in the large- $N$ limit [7-10]. The holographic description of the noncommutative gauge theories is often useful to investigate how the noncommutativity (the deformation parameter) affects the quantum properties of the gauge theories. For instance, the noncommutativity modifies the Wilson loop behavior [11-13] and glueball mass spectra [14]. The holographic duals of noncommutative gauge theories with flavor degrees of freedom have also been constructed by

Published by the American Physical Society under the terms of the Creative Commons Attribution 4.0 International license. Further distribution of this work must maintain attribution to the author(s) and the published article's title, journal citation, and DOI. Funded by SCOAP ${ }^{3}$. using probe techniques [15]. Employing the holographic description, we have been able to find that the noncommutativity is also reflected in the flavor dynamics [16]. It should be emphasized that the noncommutativity can also modify phase diagrams such as, for instance, chiral symmetry breaking in the noncommutative gauge theory $[17,18]$.

In this paper, we focus on quantum entanglement in noncommutative gauge theory. Entanglement entropy is known as a measure of entanglement in quantum systems (see, e.g., Ref. [19]). The entanglement entropy of a subsystem $A$ is defined by the von Neumann entropy of the reduced density matrix $\rho_{A}$ of the system $A$,

$$
S_{A}=-\operatorname{tr}_{A}\left(\rho_{A} \ln \rho_{A}\right) .
$$

It is possible to compute the entanglement entropy by employing the holographic approach. Ryu and Takayanagi conjectured that the holographic formula of entanglement entropy should be

$$
S_{A}=\frac{\mathcal{A}}{4 G}
$$

where $\mathcal{A}$ is the area of a minimal surface with a given boundary [20,21]. The proof of this formula was given in Ref. [22].

Quantum physics allows for a superposition of states, causing a nonlocal correlation between subsystems far apart from each other. Entanglement is the distinguishing feature of the quantum physics, including quantum field theories, and that is one of the important concepts to understand quantum aspects of the quantum physics.

It is known that entanglement entropy for nonlocal field theories whose action contains infinite derivatives follows a 
volume law, in general [23-25]. The noncommutative gauge theory is a kind of nonlocal field theory. It would be worth investigating how the nonlocality of the noncommutative gauge theory affects the properties of the entanglement entropy. The entanglement entropies in the noncommutative gauge theories have been studied based on the holographic approaches [26-29]. It has been pointed out that the divergence (cutoff dependence) part of the entanglement entropy in the large noncommutativity limit follows a volume law [26].

The holographic entanglement entropies for quantum field theories are often regularized by introducing an ultraviolet cutoff parameter. Little attention, however, has been given to the cutoff-independent part of the holographic entanglement entropies in the noncommutative gauge theories. In this paper, we try to derive the universal (cutoff-independent) part of the holographic entanglement entropy in the noncommutative Yang-Mills theory and discuss its properties. The properties of the holographic entanglement entropy in the noncommutative Yang-Mills theory should be discussed on the basis of universal (cutoff-independent) quantities.

The paper is organized as follows. In Sec. II, we introduce the holographic entanglement entropy conjectured by Ryu and Takayanagi and derive the universal (cutoffindependent) part of the holographic entanglement entropy in the noncommutative Yang-Mills theory. In Sec. III, we investigate the strong subadditivity for the holographic entanglement entropy in the noncommutative Yang-Mills theory. The properties of the mutual information that can be derived from the entanglement entropies is also discussed. In Sec. IV, we investigate the properties of the entropic $c$-function in the noncommutative Yang-Mills theory. In Sec. V, we derive the universal part of the holographic entanglement entropy in the noncommutative Yang-Mills theory at finite temperature and discuss a kind of transition based on the holographic entanglement entropy. Section VI is devoted to conclusions and discussions.

\section{HOLOGRAPHIC ENTANGLEMENT ENTROPY IN NONCOMMUTATIVE YANG-MILLS THEORY}

We consider the dual description of the noncommutative Yang-Mills theory on a spacetime $\mathbb{R}^{1+1} \times \mathbb{R}_{\theta}^{2}$, where $\mathbb{R}_{\theta}^{2}$ is the noncommutative (Moyal) plane defined by a Moyal algebra $\left[x_{2}, x_{3}\right]=i \theta$. At large $N$ and strong 't Hooft coupling, a holographic description of the noncommutative Yang-Mills theory is given by

$$
\begin{aligned}
d s^{2}= & R^{2}\left[u^{2}\left\{d x_{0}^{2}+d x_{1}^{2}+h(u)\left(d x_{2}^{2}+d x_{3}^{2}\right)\right\}\right. \\
& \left.+\left(\frac{d u^{2}}{u^{2}}+d \Omega_{5}^{2}\right)\right] \\
h= & \frac{1}{1+a^{4} u^{4}}
\end{aligned}
$$

where $R^{4}=4 \pi g_{s} N l_{s}^{4}$ and $a$ denotes the noncommutativity parameter with dimension of length.

We will use the generalized Ryu-Takayanagi formula for the ten-dimensional geometry with a varying dilaton. The holographic definition of the entanglement entropy is given by

$$
S_{A}=\frac{\mathcal{A}}{4 G_{N}^{(10)}}=\frac{1}{4 G_{N}^{(10)}} \int d^{8} \sigma e^{-2 \Phi} \sqrt{G_{\text {ind }}^{(8)}},
$$

where $G_{N}^{(10)}=8 \pi^{6} \alpha^{\prime 4}$ is the ten-dimensional Newton's constant. The five-dimensional Newton's constant $G_{N}^{(5)}$ is proportional to $G_{N}^{(10)}$ up to a volume factor $G_{N}^{(5)}=$ $G_{N}^{(10)} / \pi^{3} R^{5}$

Let us compute the entanglement entropy for an infinite strip specified by

$$
y \equiv x_{2} \in\left[-\frac{l}{2}, \frac{l}{2}\right], \quad x_{1}, x_{3} \in(-L, L),
$$

with $L \rightarrow \infty$. It is worth pointing out that when we exchange $x_{2}$ for $x_{3}$, the noncommutative deformation has no effect on the entanglement entropy. Under this configuration, the entanglement entropy defined by Eq. (2.2) takes the form

$$
S_{A}=\frac{N^{2} L^{2}}{\pi} \int d u u^{3} \sqrt{y^{\prime}(u)^{2}+\frac{1}{u^{4} h(u)}},
$$

where $y^{\prime}(u)$ is the derivative of $y$ with respect to $u$. We find that the quantity $\frac{u^{3} y^{\prime}(u)}{\sqrt{y^{2}(u)+1 / u^{4} h(u)}}$ is a constant which does not depend on $u$. This quantity leads to

$$
y^{\prime}(u)=\frac{1}{u^{2} \sqrt{h(u)}} \frac{1}{\sqrt{\frac{u^{6}}{u_{*}^{6}}-1}},
$$

where $u_{*}$ denotes an integral of motion and $u=u_{*}$ represents the point of closest approach of the extremal surface. Such surfaces have two branches, joined smoothly at $u=u_{*}$ and $u_{*}$ can be determined using the boundary conditions

$$
y\left(u_{*}\right)= \pm \frac{l}{2} .
$$

The entanglement entropy given by Eq. (2.4) at the stable solution is given by

$$
S_{A}=\frac{N^{2} L^{2}}{\pi} \int_{u_{*}}^{u_{\Lambda}} d u u^{4} \sqrt{\frac{1+(a u)^{4}}{u^{6}-u_{*}^{6}}},
$$

where $u_{\Lambda}$ is a cutoff parameter. The (dimensionless) entanglement entropy functional defined by $\mathcal{S}_{A} \equiv\left(\pi a^{2} / N^{2} L^{2}\right) S_{A}$ can be rewritten as 


$$
\mathcal{S}_{A}=\left(a u_{*}\right)^{2} \int_{u_{*} / u_{\Lambda}}^{1} \frac{d t}{t^{5}} \sqrt{\frac{t^{4}+\left(a u_{*}\right)^{4}}{1-t^{6}}},
$$

where $t \equiv u_{*} / u$ is a dimensionless variable. The ratio of the length $l$ to the noncommutativity parameter $a$ is also a function of the product of the noncommutativity parameter $a$ and the integral of motion $u_{*}$ :

$$
\frac{l}{a}=\frac{2}{a} \int_{u_{*}}^{\infty} d u y^{\prime}(u)=\frac{2}{a u_{*}} \int_{0}^{1} d t t \sqrt{\frac{t^{4}+\left(a u_{*}\right)^{4}}{1-t^{6}}}
$$

In the deep infrared bound $a u_{*} \cong 0$, we can approximate the right-hand side of Eq. (2.9) by $\frac{2}{a u_{*}} \int_{0}^{1} d t t \sqrt{\frac{t^{4}}{1-t^{6}}}$, and we have

$$
\frac{l}{a} \cong 2 \sqrt{\pi} \frac{\Gamma\left(\frac{2}{3}\right)}{\Gamma\left(\frac{1}{6}\right)} \cdot \frac{1}{a u_{*}}
$$

where $\Gamma$ denotes the gamma function. The length $l$ given by Eq. (2.10) is the same as that in the commutative $(a=0)$ version. Hereafter, we refer to the approximation in the deep infrared bound as the commutative regime. The commutative theory $(a=0)$ and the noncommutative $(a \neq 0)$ theory can be compared through the approximation in the deep infrared bound.

The entanglement entropy functional (2.8) in the commutative regime can be divided into a universal (finite) part $\mathcal{S}_{U}$ that is independent of the cutoff parameter $u_{\Lambda}$ and the divergence part $\mathcal{S}_{D}$ that depends on the cutoff parameter $u_{\Lambda}$ :

$\mathcal{S}_{U}\left(=\frac{\pi a^{2}}{N^{2} L^{2}} S_{U}\right) \cong-\frac{\sqrt{\pi}}{2} \frac{\Gamma\left(\frac{2}{3}\right)}{\Gamma\left(\frac{1}{6}\right)} \cdot\left(a u_{*}\right)^{2}$,

$\mathcal{S}_{D}\left(=\frac{\pi a^{2}}{N^{2} L^{2}} S_{D}\right) \cong \frac{1}{2} \cdot\left(a u_{\Lambda}\right)^{2}$.

In deriving this expression, we have utilized the formula

$$
\int_{0}^{1} \frac{d t}{t^{\lambda}}\left(\frac{1}{\sqrt{1-t^{\kappa}}}-1\right)-\frac{1}{\lambda-1}=\frac{\sqrt{\pi}}{\kappa} \frac{\Gamma\left(\frac{1-\lambda}{\kappa}\right)}{\Gamma\left(\frac{\kappa-2 \lambda+2}{2 \kappa}\right)},
$$

with $\lambda>1$. Notice that the finite part $\mathcal{S}_{U}$ is independent of the cutoff parameter $u_{\Lambda}$ and thus is universal quantity. Eliminating the parameter $u_{*}$ from Eqs. (2.10) and (2.11), we find the relation between the universal part $\mathcal{S}_{U}$ and the ratio $l / a$ in the commutative regime: ${ }^{1}$

\footnotetext{
${ }^{1}$ The dependence of Eq. (2.13) on the noncommutativity parameter $a$ arises from the definition of the (dimensionless) entanglement entropy functional $\mathcal{S}_{U} \equiv\left(\pi a^{2} / N^{2} L^{2}\right) S_{U}$.
}

$$
\mathcal{S}_{U}=-2\left\{\sqrt{\pi} \frac{\Gamma\left(\frac{2}{3}\right)}{\Gamma\left(\frac{1}{6}\right)}\right\}^{3}\left(\frac{l}{a}\right)^{-2} .
$$

Meanwhile, we find that the divergence part of the entanglement entropy $\mathcal{S}_{D}$ in the commutative regime is proportional to the area $L^{2}$ :

$$
\frac{2 \pi}{N^{2}} S_{D}=L^{2} \cdot u_{\Lambda}^{2} .
$$

In the deep ultraviolet bound $1 / a u_{*} \cong 0$, the ratio of the length $l$ to the noncommutativity parameter $a$ can be approximated as

$$
\frac{l}{a} \cong \frac{\sqrt{\pi}}{3} \frac{\Gamma\left(\frac{1}{3}\right)}{\Gamma\left(\frac{5}{6}\right)} \cdot a u_{*} .
$$

Hereafter, we refer to the approximation in the deep ultraviolet bound as the deep noncommutative regime [26]. The entanglement entropy functional (2.8) in the deep noncommutative regime can be divided into the universal part $\mathcal{S}_{U}$ and divergence part $\mathcal{S}_{D}$ :

$$
\mathcal{S}_{U}=\frac{\sqrt{\pi}}{24} \frac{\Gamma\left(\frac{1}{3}\right)}{\Gamma\left(\frac{5}{6}\right)} \cdot\left(a u_{*}\right)^{4}, \quad \mathcal{S}_{D}=\frac{1}{4} \cdot\left(a u_{\Lambda}\right)^{4},
$$

respectively. The relation between the finite part $\mathcal{S}_{U}$ and the ratio $l / a$ in the deep noncommutative regime is given by

$$
\mathcal{S}_{U}=\frac{1}{8}\left\{\frac{3}{\sqrt{\pi}} \frac{\Gamma\left(\frac{5}{6}\right)}{\Gamma\left(\frac{1}{3}\right)}\right\}^{3}\left(\frac{l}{a}\right)^{4}
$$

We notice that the dependence of the finite part $\mathcal{S}_{U}$ on the ratio $l / a$ is quite different between the commutative regime and the deep noncommutative regime. Eliminating the noncommutativity parameter $a$ from Eqs. (2.15) and (2.16), we find the relation between the divergence part $S_{D}$ and the length $l$ in the deep noncommutative regime:

$$
\frac{4 \pi^{3 / 2}}{3 N^{2}} \frac{\Gamma\left(\frac{1}{3}\right)}{\Gamma\left(\frac{5}{6}\right)} S_{D}=L^{2} l \cdot \frac{u_{\Lambda}^{4}}{u_{*}} .
$$

For $u_{*} \sim u_{\Lambda}$, this expression shows that the divergence part of the entanglement entropy $S_{D}$ in the deep noncommutative regime is proportional to the volume $L^{2} l$. The difference in the $l$ dependence between Eqs. (2.14) and (2.18) can be understood as the areal volume law transition [26].

The variation with $a u_{*}$ of $l / a$ is shown in Fig. 1(a). The behavior of $l / a$ is quite different between the commutative regime and the deep noncommutative regime. The length $l$ has a minimum value $l_{\min } \sim 1.614 a$ at $u_{*} \sim 0.7946 / a$ in the noncommutative theory. The ratio $l / a$ is proportional to the inverse of $a u_{*}$ for $u_{*} \ll 0.7946 / a$ and is proportional to $a u_{*}$ for $u_{*} \gg 0.7946 / a$. The ratio $l / a$ increases in the case of $a u_{*} \rightarrow \infty$ as well as in the case of $a u_{*} \rightarrow 0$. This behavior is reminiscent of the UV/IR relation [1]. 


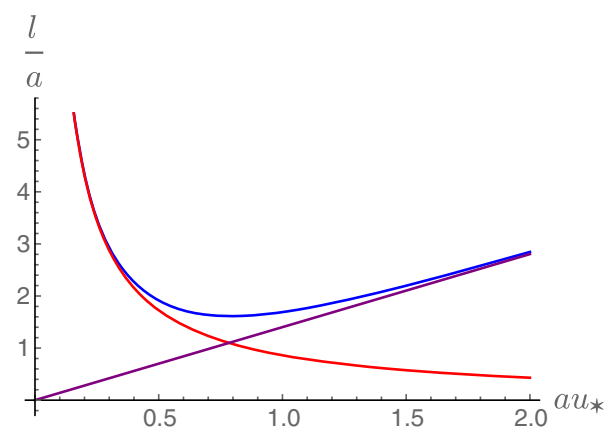

(a)

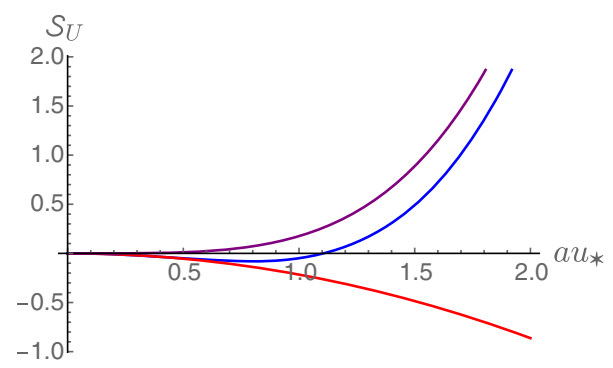

(b)

FIG. 1. (a) Variation with $a u_{*}$ of the ratio $l / a$. (b) Variation with $a u_{*}$ of the universal part $\mathcal{S}_{U}\left(=\frac{\pi a^{2}}{N^{2} L^{2}} S_{U}\right)$. The blue curve denotes the variation in the noncommutative theory. The red and purple curves denote the variation in the commutative regime and in the deep noncommutative regime, respectively.

The dimensionless quantity (2.8) can also be divided into the finite part $\mathcal{S}_{U}$ and divergence part $\mathcal{S}_{D}$ :

$$
\begin{aligned}
\mathcal{S}_{U}\left(=\frac{\pi a^{2}}{N^{2} L^{2}} S_{U}\right)= & \left(a u_{*}\right)^{2} \int_{0}^{1} d t \frac{\sqrt{t^{4}+\left(a u_{*}\right)^{4}}}{t^{5}}\left(\frac{1}{\sqrt{1-t^{6}}}-1\right) \\
& -\frac{\left(a u_{*}\right)^{2} \sqrt{1+\left(a u_{*}\right)^{4}}}{4}-\frac{1}{8} \ln \mid \frac{1+\sqrt{1+\frac{1}{\left(a u_{*}\right)^{4}}}}{1-\sqrt{1+\frac{1}{\left(a u_{*}\right)^{4}}}}, \\
\mathcal{S}_{D}\left(=\frac{\pi a^{2}}{N^{2} L^{2}} S_{D}\right)= & \frac{a^{2} u_{\Lambda}^{4}}{4 u_{*}^{2}} \sqrt{\left(\frac{u_{*}}{u_{\Lambda}}\right)^{4}+\left(a u_{*}\right)^{4}}+\frac{1}{8} \ln \mid \frac{1+\sqrt{1+\frac{1}{\left(a u_{\Lambda}\right)^{4}}}}{1-\sqrt{1+\frac{1}{\left(a u_{\Lambda}\right)^{4}}}} .
\end{aligned}
$$

In deriving the expressions above, we have utilized the formula

$$
\int d x \frac{\sqrt{x^{4}+k}}{x^{5}}=-\frac{\sqrt{x^{4}+k}}{4 x^{4}}-\frac{1}{8 \sqrt{k}} \ln \left|\frac{1+\sqrt{1+\frac{x^{4}}{k}}}{1-\sqrt{1+\frac{x^{4}}{k}}}\right|,
$$

with a constant $k$. The divergence part $\mathcal{S}_{D}$ becomes 0 when the parameter $u_{\Lambda}$ is taken to be 0 . The universal part $\mathcal{S}_{U}$ given by Eq. (2.19a) is also a function of the dimensionless quantity $a u_{*}$. The variation with $a u_{*}$ of $\mathcal{S}_{U}$ is shown in Fig. 1(b). The behavior of $\mathcal{S}_{U}$ is also different between the commutative regime and the deep noncommutative regime. The universal part $\mathcal{S}_{U}$ given by Eq. (2.19a) takes a minimum value at $u_{*} \sim 0.7946 / a$. This is the same value at which the length $l$ takes the minimum value $l_{\min }$ in the noncommutative theory.

We can evaluate the dependence of the universal part $\mathcal{S}_{U}$ on the ratio $l / a$ numerically. The variation with $l / a$ of the universal part $\mathcal{S}_{U}$ is shown in Fig. 2.

Although the sign of the universal term $\mathcal{S}_{U}$ can be negative, the sign of the entanglement entropy is positive.
As we will see later, the sign of the derivative of the universal term $\mathcal{S}_{U}$ is more important.

There is a major difference in the dependence of the universal part $\mathcal{S}_{U}$ on the ratio $l / a$ between in the commutative regime and in the deep noncommutative regime. The curve of $\mathcal{S}_{U}$ is concave downward in the commutative regime (shown as the red curve in Fig. 2), while it is concave upward in the deep noncommutative regime (shown as the purple curve in Fig. 2). This concave upward

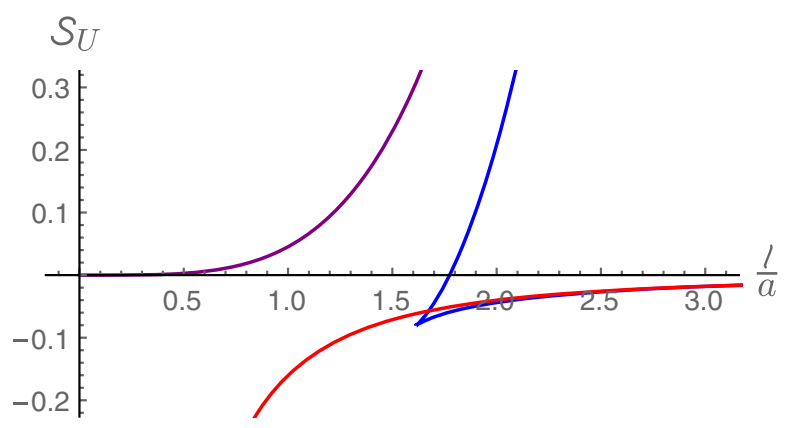

FIG. 2. The variation with $l / a$ of $\mathcal{S}_{U}$. The blue curve denotes the variation in the noncommutative theory. The red and purple curves denote the variation in the commutative regime and in the deep noncommutative regime, respectively. 
curve suggests that the behavior of $\mathcal{S}_{U}$ in the deep noncommutative regime becomes unphysical. In the noncommutative theory, the curve of $\mathcal{S}_{U}$ branches into a concave downward curve and a concave upward curve at $l / a=$ $l_{\min } / a$ (shown as the blue curves in Fig. 2). Figure 2 shows that the blue concave downward curve (lower branch) and the blue concave upward curve (upper branch) asymptotically approach the red concave downward curve and the purple concave upward curve in the limit $l / a \rightarrow \infty$, respectively.

We see that the universal part $\mathcal{S}_{U}$ with the identical ratio $l / a\left(>l_{\min } / a\right)$ actually has different values in the noncommutative theory. Therefore, the concave downward curve becomes presumably dominated for the curve of $\mathcal{S}_{U}$ in the noncommutative theory. When the concave upward curve of $\mathcal{S}_{U}$ is realized in the noncommutative theory, the derivative of the universal part $\mathcal{S}_{U}$ with respect to the ratio $l / a$ seems to be discontinuous at the point $l / a=l_{\min } / a$. This behavior could be interpreted as the areal volume law transition [26] from the viewpoint of the universal part $\mathcal{S}_{U}$.

\section{STRONG SUBADDITIVITY AND MUTUAL INFORMATION}

Entanglement entropies are subject to an inequality known as strong subadditivity [30,31]. This inequality
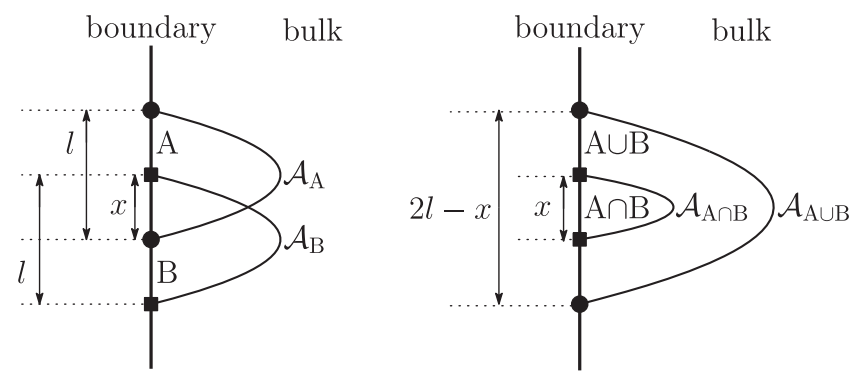

FIG. 3. Left: two overlapping regions $\mathrm{A}$ and $\mathrm{B}$ of the boundary, with their respective minimal bulk hypersurfaces $\mathcal{A}_{\mathrm{A}}$ and $\mathcal{A}_{\mathrm{B}}$. Right: two regions $\mathrm{A} \cup \mathrm{B}$ and $\mathrm{A} \cap \mathrm{B}$ of the boundary, with their respective minimal hypersurfaces $\mathcal{A}_{\mathrm{A \cup B}}$ and $\mathcal{A}_{\mathrm{A} \cap \mathrm{B}}$.

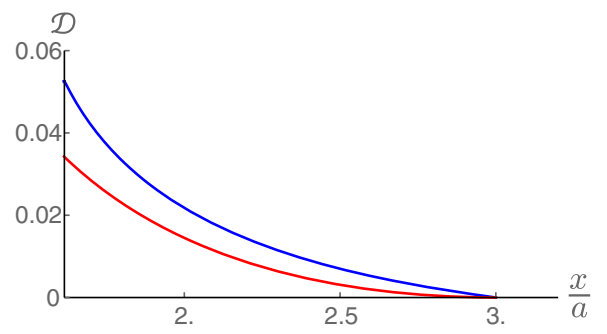

(a) can be stated as $S(A)+S(B) \geq S(A \cup B)+S(A \cap B)$ for any two regions of space $A$ and $B$. We would like to examine if the holographic entanglement entropies in the noncommutative Yang-Mills theory are subject to the strong subadditivity. We consider two identical infinite strips with a width $l$, arranged to overlap each other by a length $x$ along the $x^{2}$ direction. A conceptual figure is given in Fig. 3.

Let us define the following quantity:

$$
D(x)=2 S_{U}\left(\frac{l}{a}\right)-S_{U}\left(\frac{x}{a}\right)-S_{U}\left(\frac{2 l-x}{a}\right) .
$$

The quantity $\mathcal{D}=\frac{\pi a^{2}}{N^{2} L^{2}} D$ becomes positive when the strong subadditivity inequality is satisfied. The variation of $d$ with $x / a$ is shown in Fig. 4.

As we expected, the strong subadditivity inequality for the entanglement entropies in the commutative regime is satisfied. On the other hand, the strong subadditivity inequality for the entanglement entropies in the deep noncommutative regime is not satisfied. The strong subadditivity inequality for the entanglement entropies in the noncommutative theory is also satisfied under the condition $u_{*}<0.7946 / a$. It is interesting to note that the strong subadditivity inequality for the entanglement entropies in the noncommutative theory is partially satisfied under the condition $u_{*}>0.7946 / a$, in spite of their unphysical behavior. We find that the inequality in the noncommutative theory is completely satisfied when the two subsystems almost overlap.

The mutual information $I(A, B)$ of any two regions of space $A$ and $B$ can be written in terms of the entanglement entropies as $I(A, B)=S(A)+S(B)-S(A \cup B)$. It measures how much we learn about one region of space $A$ by observing the other region of space $B$. This interpretation convinces us that the mutual information $I(A, B)$ has to be non-negative. The non-negativity property of the mutual information is guaranteed by the subadditivity of the entanglement entropy. We would like to examine if the mutual information in the noncommutative Yang-Mills theory is subject to the non-negativity property. We consider two infinite strips, each of length $l$, separated by a

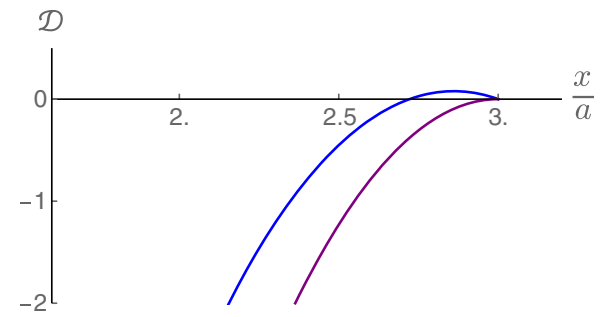

(b)

FIG. 4. (a) Variation with $x / a$ of the quantity $\mathcal{D}$ for $l / a=3$ and $u_{*}<0.7946 / a$. (b) Variation of the quantity $\mathcal{D}$ with $x / a$ for $l / a=3$ and $u_{*}>0.7946 / a$. The blue curve denotes the variation in the noncommutative theory. The red and purple curves denote the variation in the commutative regime and in the deep noncommutative regime, respectively. 

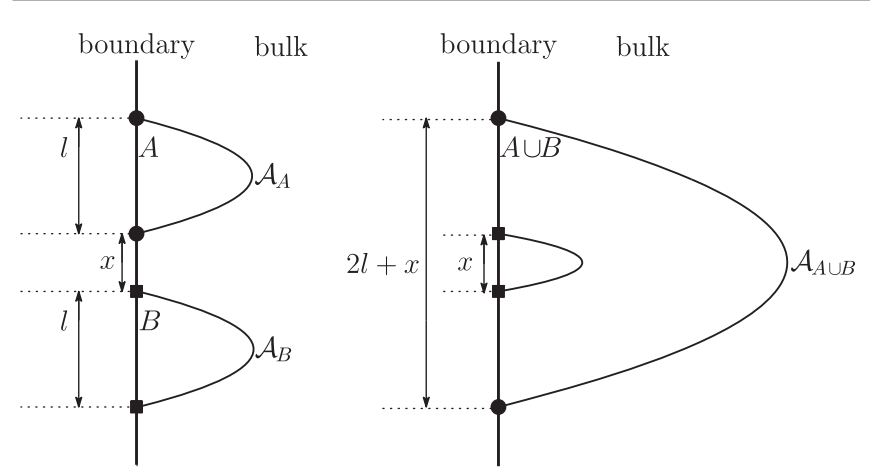

FIG. 5. Left: two separated regions $\mathrm{A}$ and $\mathrm{B}$ of the boundary, with their respective minimal bulk hypersurfaces $\mathcal{A}_{\mathrm{A}}$ and $\mathcal{A}_{\mathrm{B}}$. Right: the region $\mathrm{A} \cup \mathrm{B}$ of the boundary, with their respective minimal hypersurface $\mathcal{A}_{\mathrm{A} \cup \mathrm{B}}$.

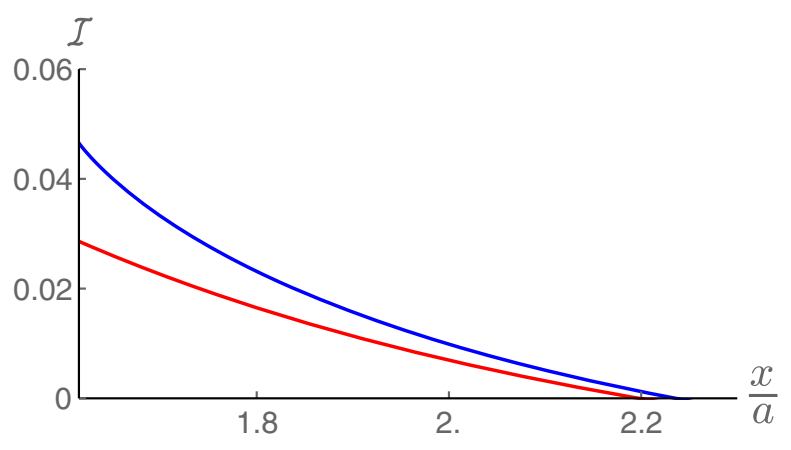

FIG. 6. Variation with $x / a$ of the quantity $\mathcal{I}$ for $l / a=3$ and $u_{*}<0.7946 / a$. The blue curve denotes the variation in the noncommutative theory. The red curve denotes the variation in the commutative regime.

distance $x$ along the $x^{2}$ direction. A conceptual figure is given in Fig. 5.

Let us define the following quantity:

$$
I(x)=2 S_{U}\left(\frac{l}{a}\right)-S_{U}\left(\frac{x}{a}\right)-S_{U}\left(\frac{2 l+x}{a}\right) .
$$

The variation of the quantity $\mathcal{I}=\frac{\pi a^{2}}{N^{2} L^{2}} I$ with $x / a$ is shown in Fig. 6.

We find that the value of the mutual information in the noncommutative theory under the condition $u_{*}<0.7946 / a$ is slightly larger than that in the commutative regime. The mutual information in the noncommutative theory under the condition $u_{*}>0.7946 / a$ (and also in the deep noncommutative regime) takes a negative value for all values of the distance $x$.

\section{ENTROPIC $c$-FUNCTION}

It is well known that there exists a so-called $c$-function that is a positive real function and is monotonically decreasing under the renormalization group flow. The $c$-function can be defined by means of the entanglement

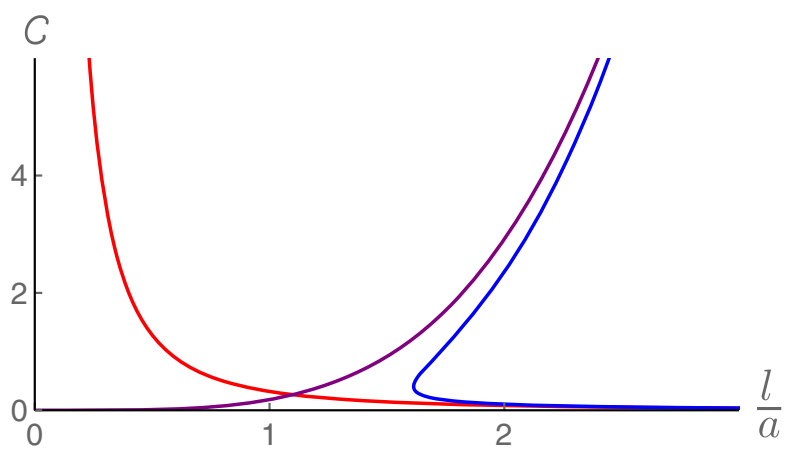

FIG. 7. Variation with $l / a$ of $\mathcal{C}$. The blue curve denotes the variation in the noncommutative theory. The red and purple curves denote the variation in the commutative regime and in the deep noncommutative regime, respectively.

entropy, and this quantity is called entropic $c$-function [32-34]. For an infinite strip subsystem with length $l$, the entropic $c$-function denoted by $C$ can be rewritten as $[26,35,36]$

$$
C(l)=\frac{d S_{A}}{d \ln l}=l \frac{d S_{A}}{d l} .
$$

Note that this quantity does not depend on the cutoff parameter $u_{\Lambda}$. The variation of $\mathcal{C}(l)=\frac{\pi a^{2}}{N^{2} L^{2}} C(l)$ with $l / a$ is shown in Fig. 7.

Since the $c$-function denoted by $\mathcal{C}(l)$ measures the number of degrees of freedom, it is expected to satisfy the inequality $\mathcal{C}\left(l_{\mathrm{UV}}\right) \geq \mathcal{C}\left(l_{\mathrm{IR}}\right)\left(\right.$ for $\left.l_{\mathrm{UV}} \leq l_{\mathrm{IR}}\right)$ and the derivative of $\mathcal{C}$ with respect to $l$ is expected to be negative. As we expected, the entropic $c$-function in the commutative regime (shown as the red curve in Fig. 7) satisfies such properties. The entropic $c$-function in the deep noncommutative regime (shown as the purple curve in Fig. 7), however, does not follow the expected behavior. It diverges as $l$ approaches $\infty$. The entropic $c$-function in the noncommutative theory (shown as the blue curve in Fig. 7) under the condition $u_{*}<0.7946 / a$ also satisfies the inequality $\mathcal{C}\left(l_{\mathrm{UV}}\right) \geq \mathcal{C}\left(l_{\mathrm{IR}}\right)$ ( for $\left.l_{\mathrm{UV}} \leq l_{\mathrm{IR}}\right)$ and the derivative of $\mathcal{C}$ with respect to $l$ is negative. The turning point on the blue curves in Figs. 2 and 7 can be observed at the same value for $l / a\left(=l_{\min } / a\right)$.

The behavior of the entropic $c$-function in the commutative regime under the condition $l \rightarrow 0$ is similar to that in the deep noncommutative regime under the condition $l \rightarrow \infty$. This phenomenon also seems to be relevant to the UV/IR mixing phenomenon [1], in the sense that the UV divergence of the commutative regime appears to be replaced by the IR singularity of the deep noncommutative regime.

\section{FINITE TEMPERATURE}

In this section, we consider the holographic entanglement entropy in the noncommutative Yang-Mills theory at 
finite temperature. A holographic description of the noncommutative Yang-Mills theory at finite temperature is given by

$$
\begin{aligned}
d s^{2}= & R^{2}\left[u^{2}\left\{f(u) d x_{0}^{2}+d x_{1}^{2}+h(u)\left(d x_{2}{ }^{2}+d x_{3}{ }^{2}\right)\right\}\right. \\
& \left.+\left(\frac{d u^{2}}{u^{2} f(u)}+d \Omega_{5}^{2}\right)\right] \\
f= & 1-\left(\frac{u_{T}}{u}\right)^{4}
\end{aligned}
$$

where $u_{T}$ is a parameter with dimension of mass and is the lower bound of $u$. The corresponding temperature $T$ of the background can be obtained to be $T=u_{T} / \pi$.

We compute the entanglement entropy for an infinite strip specified by Eq. (2.3) with $L \rightarrow \infty$. Under this configuration, the entanglement entropy denoted by $S_{A T}$ at the stable solution is modified to include the parameter $u_{T}$ :

$$
S_{A T}=\frac{\mathcal{A}}{4 G_{N}^{(10)}}=\frac{N^{2} L^{2}}{\pi} \int_{u_{*}}^{u_{\Lambda}} d u u^{6} \sqrt{\frac{1+(a u)^{4}}{\left(u^{6}-u_{*}^{6}\right)\left(u^{4}-u_{T}^{4}\right)}} .
$$

The length $l$ is also modified to include the parameter $u_{T}$ :

$$
\frac{l}{2}=u_{*}^{2} \int_{u_{*}}^{\infty} d u \sqrt{\frac{1+(a u)^{4}}{\left(u^{6}-u_{*}^{6}\right)\left(u^{4}-u_{T}^{4}\right)}} .
$$

We can find that in the large- $l$ limit, the main contribution of (the finite part of) the integrals (5.2) and (5.3) coming from the region near $u \sim u_{*} \sim u_{T}$ leads to the relation

$$
S_{A T}^{\text {finite }}=\frac{\pi^{2}}{2} N^{2} T^{3} \cdot L^{2} l
$$

The entanglement entropy given by Eq. (5.4) is proportional to the volume $L^{2} l$. Thus, it is extensive as in the thermal entropy. The $l$ dependence of the entanglement entropy shown by Eq. (5.4) is at first glance similar to that shown by Eq. (2.18). It should be noted, however, that Eq. (2.18) represents the dependence of the divergent part of the entanglement entropy, while Eq. (5.4) represents the dependence of the finite part of the entanglement entropy.

The (dimensionless) entanglement entropy functional $\mathcal{S}_{A T}=\left(\pi a^{2} / N^{2} L^{2}\right) S_{A T}$ can be rewritten as

$$
\mathcal{S}_{A T}=\left(a u_{*}\right)^{2} \int_{u_{*} / u_{\Lambda}}^{1} \frac{d t}{t^{5}} \sqrt{\frac{t^{4}+\left(a u_{*}\right)^{4}}{\left(1-t^{6}\right)\left(1-\left(\frac{\tau t}{a u_{*}}\right)^{4}\right)}},
$$

where $t \equiv u_{*} / u$ and $\tau$ is a dimensionless parameter defined by $\tau \equiv a u_{T}$. The ratio of the length $l$ to the noncommutativity parameter $a$ is also modified to include the parameter $\tau$ :

$$
\frac{l}{a}=\frac{2}{a u_{*}} \int_{0}^{1} d t t \sqrt{\frac{t^{4}+\left(a u_{*}\right)^{4}}{\left(1-t^{6}\right)\left(1-\left(\frac{\tau t}{a u_{*}}\right)^{4}\right)}} .
$$

Equation (5.4) can also be rewritten in terms of $\mathcal{S}_{A T}^{\text {finite }}=\left(\pi a^{2} / N^{2} L^{2}\right) S_{A T}^{\text {finite }}, l / a$, and $\tau$ :

$$
\mathcal{S}_{A T}^{\text {finite }}=\tau^{3} \cdot \frac{l}{a} .
$$

The entanglement entropy functional given by Eq. (5.5) can be divided into the universal part $\mathcal{S}_{U T}$ and divergence part $\mathcal{S}_{D T}$ :

$$
\begin{aligned}
\mathcal{S}_{U T}= & \left(a u_{*}\right)^{2} \int_{0}^{1} d t \frac{\sqrt{t^{4}+\left(a u_{*}\right)^{4}}}{t^{5}} \\
& \times\left\{\frac{1}{\sqrt{\left(1-t^{6}\right)\left(1-\left(\frac{\tau t}{a u_{*}}\right)^{4}\right)}}-1\right\} \\
& -\frac{\left(a u_{*}\right)^{2} \sqrt{1+\left(a u_{*}\right)^{4}}}{4}-\frac{1}{8} \ln \mid \frac{1+\sqrt{1+\frac{1}{\left(a u_{*}\right)^{4}}}}{1-\sqrt{1+\frac{1}{\left(a u_{*}\right)^{4}}}},
\end{aligned}
$$

$$
\mathcal{S}_{D T}=\frac{a^{2} u_{\Lambda}^{4}}{4 u_{*}^{2}} \sqrt{\left(\frac{u_{*}}{u_{\Lambda}}\right)^{4}+\left(a u_{*}\right)^{4}}+\frac{1}{8} \ln \left|\frac{1+\sqrt{1+\frac{1}{\left(a u_{\Lambda}\right)^{4}}}}{1-\sqrt{1+\frac{1}{\left(a u_{\Lambda}\right)^{4}}}}\right| .
$$

Although the universal part $\mathcal{S}_{U T}$ depends on the parameter $\tau$, related to the temperature $T$, the divergence part $\mathcal{S}_{D T}$ does not depend on the parameter $\tau$. This fact indicates that the behavior of the universal part of the entanglement entropy is modified at finite temperature, while the behavior of the divergent part of the entanglement entropy is not modified at all.

We can also evaluate the dependence of the universal part of the entanglement entropy functional $S_{U T}$ given by Eq. (5.8a) on the length $l$ given by Eq. (5.6) numerically. The variation with $l / a$ of the universal part $\mathcal{S}_{U T}$ is shown in Fig. 8.

Here, $\tau=0$ corresponds to the zero-temperature case discussed in the previous sections. (It should be noted that the domain and range of the graph in Fig. 8 are different from that in Fig. 2.)

Notice that the minimum length $l_{\min }$ exists even at finite temperature. It can be found that the value of $l_{\min }$ increases with increasing temperature $\tau$. The change in the minimum length $l_{\min }$ with temperature $\tau$ is however slight. There are no significant changes in the $l$ dependence of the entanglement entropy in the region $a u_{*}>\tau\left(u_{*}>u_{T}\right)$. In contrast, Fig. 8(b) shows that the relationship expressed in 


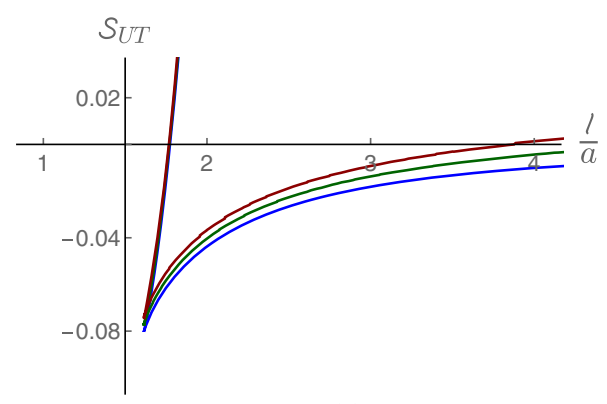

(a)

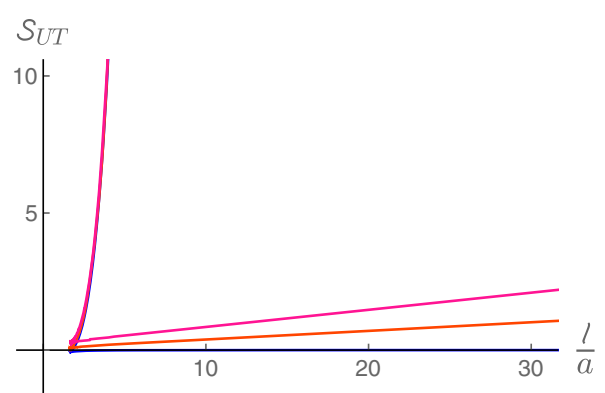

(b)

FIG. 8. Variation with $l / a$ of $\mathcal{S}_{U T}$ in the noncommutative theory. (a) The blue, green, and brown curves correspond, respectively, to $\tau=0.00,0.16$, and 0.19. (b) The blue, orange, and magenta curves correspond, respectively, to $\tau=0.00,0.40$, and 0.50 .

Eq. (5.7) is approximately satisfied between $\mathcal{S}_{U T}$ and $l / a$ in the large- $l$ limit (in the region $a u_{*} \sim \tau$ or $u_{*} \sim u_{T}$ ).

Generally, there is another surface that satisfies the boundary condition (2.6) because the holographic dual of the field theory at finite temperature involves black hole horizons [36]. The surface is parametrized as

$$
y=x_{2}= \pm \frac{l}{2}, \quad u=u_{T}
$$

We call this a piecewise smooth surface, to distinguish it from the smooth minimal surface. Therefore, there are two candidates for the surfaces to which Ryu-Takayanagi prescription can be applied, the smooth minimal surface and the piecewise smooth surface. Let us compute the area of the piecewise smooth surface denoted by $\mathcal{A}^{\prime}$ and examine the behavior of two areas $\mathcal{A}$ and $\mathcal{A}^{\prime}$ as a function of the ratio $l / a$. The induced line elements for different segments are

$$
\begin{aligned}
\frac{d s^{2}}{R^{2}}= & u^{2}\left\{f(u) d x_{0}{ }^{2}+d x_{1}{ }^{2}+h(u) d x_{3}{ }^{2}\right\} \\
& +\frac{d u^{2}}{u^{2} f(u)}+d \Omega_{5}{ }^{2} \quad \text { for } y= \pm \frac{l}{2} \\
\frac{d s^{2}}{R^{2}}= & u^{2}\left\{f(u) d x_{0}{ }^{2}+d x_{1}{ }^{2}+h\left(u_{T}\right)\left(d x_{2}{ }^{2}+d x_{3}{ }^{2}\right\}\right. \\
& +d \Omega_{5}{ }^{2} \text { for } \quad \text { for } u=u_{T} .
\end{aligned}
$$

The entanglement entropy denoted by $S_{A T}^{\prime}$ is then given by

$S_{A T}^{\prime}=\frac{\mathcal{A}^{\prime}}{4 G_{N}^{(10)}}=\frac{N^{2} L^{2}}{\pi}\left\{\int_{u_{*}}^{u_{\Lambda}} d u u^{3} \sqrt{\frac{1+(a u)^{4}}{u^{4}-u_{T}^{4}}}+\frac{l}{2} u_{T}^{3}\right\}$.

The (dimensionless) entanglement entropy functional $\mathcal{S}_{A T}^{\prime}=\left(\pi a^{2} / N^{2} L^{2}\right) S_{A T}^{\prime}$ can also be divided into the universal part $\mathcal{S}_{U T}^{\prime}$ and divergence part $\mathcal{S}_{D T}^{\prime}$ :

$$
\begin{aligned}
\mathcal{S}_{U T}^{\prime}= & \left(a u_{*}\right)^{2} \int_{0}^{1} d t \frac{\sqrt{t^{4}+\left(a u_{*}\right)^{4}}}{t^{5}}\left\{\frac{1}{\sqrt{1-\left(\frac{\tau t}{a u_{*}}\right)^{4}}}-1\right\} \\
& +\frac{\tau^{3}}{a u_{*}} \int_{0}^{1} d t \sqrt{\frac{t^{4}+\left(a u_{*}\right)^{4}}{\left(1-t^{6}\right)\left(1-\left(\frac{\tau t}{a u_{*}}\right)^{4}\right)}} \\
& -\frac{\left(a u_{*}\right)^{2} \sqrt{1+\left(a u_{*}\right)^{4}}}{4}-\frac{1}{8} \ln \mid \frac{1+\sqrt{1+\frac{1}{\left(a u_{*}\right)^{4}}} \mid}{1-\sqrt{1+\frac{1}{\left(a u_{*}\right)^{4}}} \mid} \\
\mathcal{S}_{D T}^{\prime}= & \frac{a^{2} u_{\Lambda}^{4}}{4 u_{*}^{2}} \sqrt{\left(\frac{u_{*}}{u_{\Lambda}}\right)^{4}+\left(a u_{*}\right)^{4}}+\frac{1}{8} \ln \left|\frac{1+\sqrt{1+\frac{1}{\left(a u_{\Lambda}\right)^{4}}}}{1-\sqrt{1+\frac{1}{\left(a u_{\Lambda}\right)^{4}}}}\right|
\end{aligned}
$$

The divergence part $\mathcal{S}_{D T}^{\prime}$ is the same as $\mathcal{S}_{D T}$, and thus $\mathcal{S}_{D T}^{\prime}$ does not depend on the parameter $\tau$. The variation with $l / a$ of the universal part $\mathcal{S}_{U T}$ and $\mathcal{S}_{U T}^{\prime}$ is shown in Fig. 9.

We would like to focus on the behavior of the curves of the entanglement entropy functionals $\mathcal{S}_{U T}$ and $\mathcal{S}_{U T}^{\prime}$ near $a u_{*} \sim \tau\left(u_{*} \sim u_{T}\right)$. As shown in Fig. 9, the value of the entanglement entropy functional $\mathcal{S}_{U T}^{\prime}$ also increases with

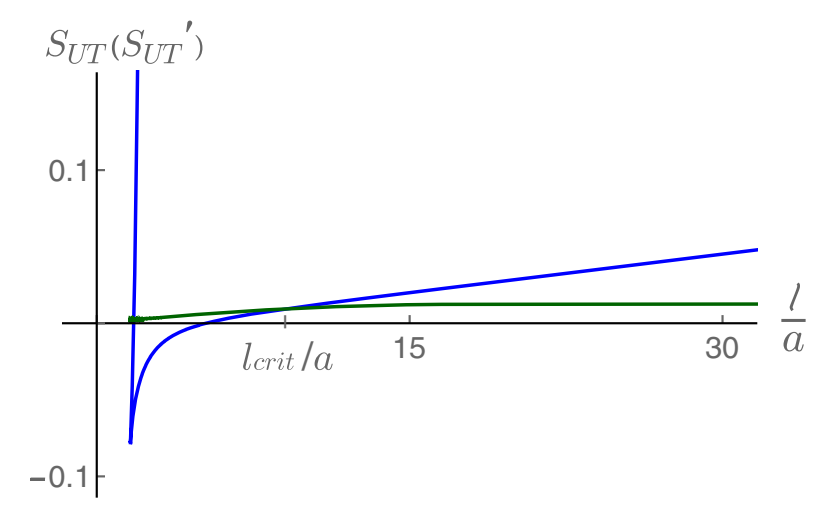

FIG. 9. Variation with $l / a$ of $\mathcal{S}_{U T}$ (blue curve) and $\mathcal{S}_{U T}{ }^{\prime}$ (green curve) in the noncommutative theory. Both the blue and green curves correspond to $\tau=0.15$. 


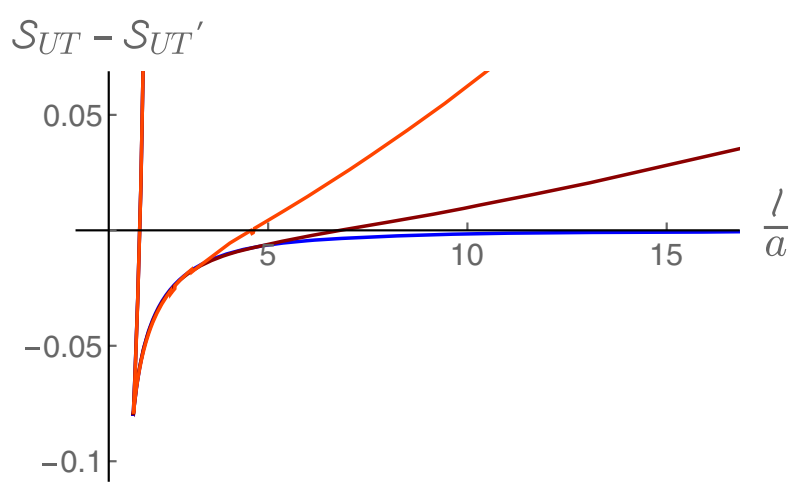

FIG. 10. Variation with $l / a$ of the difference $\mathcal{S}_{U T}-\mathcal{S}_{U T}{ }^{\prime}$. The blue, brown, and orange curves correspond, respectively, to $\tau=0.00,0.20$, and 0.30 .

the increase of $l / a$. As is clear from the fact that the slope of the curve of $\mathcal{S}_{U T}^{\prime}$ is almost flat, however, the increase rate of $\mathcal{S}_{U T}^{\prime}$ with respect to $l / a$ is smaller than that of $\mathcal{S}_{U T}$. Therefore, the curves of the entanglement entropies $\mathcal{S}_{U T}$ and $\mathcal{S}_{U T}^{\prime}$ cross at $l=l_{\text {crit }}>l_{\min }$. This fact shows that the entanglement entropy is governed by the configuration of the piecewise smooth surface for $l>l_{\text {crit }}$, since the RyuTakayanagi prescription requires the selection of curved surfaces with a smaller area. In other words, there is a transition for the entanglement entropy in the noncommutative theory. It has been shown that such transitions do not occur in the corresponding (four-dimensional) commutative theory [36].

The variation with $l / a$ of the difference $\mathcal{S}_{U T}-\mathcal{S}_{U T}^{\prime}$ is shown in Fig. 10.

$\mathcal{S}_{U T}-\mathcal{S}_{U T}^{\prime}=0$ corresponds to the intersection of curves of the entanglement entropies $\mathcal{S}_{U T}$ and $\mathcal{S}_{U T}^{\prime}$. Figure 10 shows that the transition for the entanglement entropy occurs at positive $\tau$, whereas the transition for the entanglement entropy does not occur at $\tau=0$. The configuration of the smooth surface has the lowest entanglement entropy for small $\tau$ and is the dominant contribution. On the other hand, the configuration of the piecewise smooth surface has the lowest entanglement entropy for large $\tau$ and becomes the dominant contribution.

\section{CONCLUSIONS AND DISCUSSIONS}

In this paper, we have examined the properties of the holographic entanglement entropy in the holographic dual of the noncommutative Yang-Mills theory. The finite part of the holographic entanglement entropy in the noncommutative Yang-Mills theory can be derived without cutoff dependence, and thus is universal. Although the divergence part of the holographic entanglement entropy in the commutative regime $\left(a u_{*} \rightarrow 0\right.$ limit) follows the area law, in the deep noncommutative regime $\left(a u_{*} \rightarrow \infty\right.$ limit $)$ it follows the volume law. This area/volume law transition [26] could be understood as a feature of nonlocal field theories.
The universal part of the holographic entanglement entropy as a function of length $l$ in the noncommutative theory exhibits a peculiar behavior. There exists a minimum length $l_{\min }$ in the noncommutative theory, and the curve of the entanglement entropy branches at points of the minimum length $l_{\min }$. This behavior seems to be a remarkable feature that somehow reflects the area/volume law transition.

The holographic entanglement entropy in the deep noncommutative regime does not satisfy the strong subadditivity inequality. In addition, the value of the mutual information of any two regions of space in the deep noncommutative regime takes a negative value for all values of the distance. These undesired results might be related to the nonlocal properties of the noncommutative Yang-Mills theory. These points require further discussion. It should be emphasized that the noncommutativity of space itself does not violate the strong subadditivity. The value of the mutual information in the noncommutative theory under the condition $a u_{*}<0.7946$ takes a rather large value compared to that in the commutative regime.

The entropic $c$-function in the noncommutative theory does not satisfy monotonicity with respect to $l$ as the scale parameter. The monotonicities of the $c$-functions built from the entanglement entropy are derived as a result of the strong subadditivity and Lorentz invariance of the theory. This behavior of the entropic $c$-function in the noncommutative theory might be understood from the breaking of Lorentz symmetry. On the other hand, the behavior of the entropic $c$-function in the IR limit of the commutative regime is similar to that in the UV limit of the deep noncommutative regime, if we interpret $l \rightarrow \infty$ as the infrared limit and $l \rightarrow 0$ as the ultraviolet limit. This phenomenon seems to be a kind of UV/IR relation. It would be interesting to discuss such arguments with the entropic $c$-theorems in four dimensions ( $a$-theorem) [37,38].

There exists a minimum length $l_{\min }$ that gives a branch point of the curve of the entanglement entropy even in the noncommutative theory at finite temperature. On the other hand, the effect of temperature on the behavior of the entanglement entropy curve becomes remarkable for large $l / a\left(u_{*} \sim u_{T}\right)$. We also notice that there is a transition from the configuration of the smooth surface to the piecewise smooth surface for the entanglement entropy in the noncommutative theory. The anisotropy of the noncommutativity is considered to be a major factor in inducing this transition.

The holographic entanglement entropy can act as an order parameter for the confinement/deconfinement phase in a confining gauge theory and can be used as a diagnostic tool to examine the confinement/deconfinement phase structure ${ }^{2}[35,40]$. It is interesting to discuss how the noncommutativity parameter affects the confinement/ deconfinement phase structure. In addition, it has been

\footnotetext{
${ }^{2}$ It was also pointed out that the entanglement measures may be unable to discriminate confining theories from nonconfining ones with a mass gap [39].
} 
known that the existence of a "stripe phase," characterized by the peculiar position dependence of the order parameter in the noncommutative scalar field theories [41,42]. It seem that such phases do not yet manifest in the holographic entanglement entropy of the noncommutative Yang-Mills theory. We hope to discuss these subjects in the future.
[1] S. Minwalla, M. V. Raamsdonk, and N. Seiberg, Noncommutative perturbative dynamics, J. High Energy Phys. 02 (2000) 020.

[2] A. Armoni, Comments on perturbative dynamics of noncommutative Yang-Mills theory, Nucl. Phys. B593, 229 (2001).

[3] A. Connes, M. R. Douglas, and A. Schwarz, Noncommutative geometry and matrix theory: Compactification on tori, J. High Energy Phys. 02 (1998) 003.

[4] M. R. Douglas and C. Hull, D-branes and the Noncommutative Torus, J. High Energy Phys. 02 (1998) 008.

[5] F. Ardalan, H. Arfaei, and M. M. Sheikh-Jabbari, Noncommutative geometry from strings and branes, J. High Energy Phys. 02 (1999) 016.

[6] N. Seiberg and E. Witten, String theory and noncommutative geometry, J. High Energy Phys. 09 (1999) 032.

[7] A. Hashimoto and N. Itzhaki, Non-commutative Yang-Mills and the AdS/CFT correspondence, Phys. Lett. B 465, 142 (1999).

[8] J. M. Maldacena and J. G. Russo, Large N limit of noncommutative gauge theories, J. High Energy Phys. 09 (1999) 025.

[9] M. Alishahiha, Y. Oz, and M. M. Sheikh-Jabbari, Supergravity and large $\mathrm{N}$ noncommutative field theories, J. High Energy Phys. 11 (1999) 007.

[10] M. Li and Y.-S. Wu, Holography and Noncommutative Yang-Mills Theory, Phys. Rev. Lett. 84, 2084 (2000).

[11] A. Dhar and Y. Kitazawa, Wilson loops in strongly coupled noncommutative gauge theories, Phys. Rev. D 63, 125005 (2001).

[12] S. Lee and S. Sin, Wilson loop and dimensional reduction in non-commutative gauge theories, Phys. Rev. D 64, 086002 (2001).

[13] H. Takahashi, T. Nakajima, and K. Suzuki, D1/D5 system and Wilson loops in (Non-)commutative gauge theories, Phys. Lett. B 546, 273 (2002).

[14] T. Nakajima, K. Suzuki, and H. Takahashi, Glueball mass spectra for supergravity duals of noncommutative gauge theories, J. High Energy Phys. 01 (2006) 016.

[15] D. Arean, A. Paredes, and A. V. Ramallo, Adding flavor to the gravity dual of non-commutative gauge theories, J. High Energy Phys. 08 (2005) 017.

[16] T. Nakajima, Y. Ohtake, and K. Suzuki, The spectrum of low spin mesons at finite temperature in holographic noncommutative QCD, Int. J. Mod. Phys. A 28, 1350171 (2013).

[17] T. Nakajima, Y. Ohtake, and K. Suzuki, Chiral symmetry restoration in holographic noncommutative QCD, J. High Energy Phys. 09 (2011) 054.
[18] T. Nakajima, Y. Ohtake, and K. Suzuki, Baryon number current in holographic noncommutative QCD, Phys. Rev. D 96, 046018 (2017).

[19] P. Calabrese and J. Cardy, Entanglement entropy and quantum field theory: A non-technical introduction, Int. J. Quantum. Inform. 04, 429 (2006).

[20] S. Ryu and T. Takayanagi, Holographic Derivation of Entanglement Entropy from AdS/CFT, Phys. Rev. Lett. 96, 181602 (2006).

[21] S. Ryu and T. Takayanagi, Aspects of holographic entanglement entropy, J. High Energy Phys. 08 (2006) 045.

[22] L. Aitor and J. Maldacena, Generalized gravitational entropy, J. High Energy Phys. 08 (2013) 090.

[23] N. Shiba and T. Takayanagi, Volume law for the entanglement entropy in non-local QFTs, J. High Energy Phys. 02 (2014) 033.

[24] U. Kol, C. Nunez, D. Schofield, J. Sonnenschein, and M. Warschawski, Confinement, phase transitions and nonlocality in the entanglement entropy, J. High Energy Phys. 06 (2014) 005.

[25] D.-W. Pang, On holographic entanglement entropy of nonlocal field theories, Phys. Rev. D 89, 126005 (2014).

[26] J. L. F. Barbon and C. A. Fuertes, Holographic entanglement entropy probes (non)locality, J. High Energy Phys. 04 (2008) 096.

[27] W. Fischler, A. Kundu, and S. Kundu, Holographic entanglement in a noncommutative gauge theory, J. High Energy Phys. 01 (2014) 137.

[28] J. L. Karczmarek and C. Rabideau, Holographic entanglement entropy in nonlocal theories, J. High Energy Phys. 10 (2013) 078.

[29] T. Jia and Z. Xu, Noncommutativity and holographic entanglement entropy, arXiv:1612.04857.

[30] E. H. Lieb and M. B. Ruskai, Proof of the strong subadditivity of quantum-mechanical entropy, J. Math. Phys. (N.Y.) 14, 1938 (1973).

[31] E. H. Lieb and M. B. Ruskai, A Fundamental Property of Quantum-Mechanical Entropy, Phys. Rev. Lett. 30, 434 (1973).

[32] H. Casini and M. Huerta, A finite entanglement entropy and the c-theorem, Phys. Lett. B 600, 142 (2004).

[33] H. Casini, C. D. Fosco, and M. Huerta, Entanglement and alpha entropies for a massive Dirac field in two dimensions, J. Stat. Mech. (2005) P07007.

[34] H. Casini and M. Huerta, Entanglement and alpha entropies for a massive scalar field in two dimensions, J. Stat. Mech. (2005) P12012.

[35] I. R. Klebanov, D. Kutasov, and A. Murugan, Entanglement as a probe of confinement, Nucl. Phys. B796, 274 (2008). 
[36] I. Bah, A. Faraggi, L. A. Pando Zayas, and C. A. TerreroEscalante, Holographic entanglement entropy at finite temperature, Int. J. Mod. Phys. A 24, 2703 (2009).

[37] S. N. Solodukhin, The a-theorem and entanglement entropy. arXiv:1304.4411.

[38] H. Casini, E. Teste, and G. Torroba, Markov Property of the CFT Vacuum and the a-Theorem, Phys. Rev. Lett. 118, 261602 (2017).

[39] N. Jokela and J. G. Subils, Is entanglement a probe of confinement?, J. High Energy Phys. 02 (2021) 147.
[40] T. Nishioka and T. Takayanagi, AdS bubbles, entropy and closed string tachyons, J. High Energy Phys. 01 (2007) 090.

[41] S. S. Gubser and S. L. Sondhi, Phase structure of noncommutative scalar field theories, Nucl. Phys. B605, 395 (2001).

[42] P. Castorina and D. Zappala, Spontaneous breaking of translational invariance in non-commutative lambda $\phi^{4}$ theory in two dimensions, Phys. Rev. D 77, 027703 (2008). 